\title{
Review adverts for botulinum toxin to ensure compliance, dentists warned
}

The Dental Defence Union and the Faculty of General Dental Practice (FGDP(UK)) have both encouraged dentists and dental practices offering botulinum toxin injections to ensure their marketing is legally compliant.

Advertising prescription-only medicines (POMs) to the public breaches the Human Medicines Regulations 2012 as well as

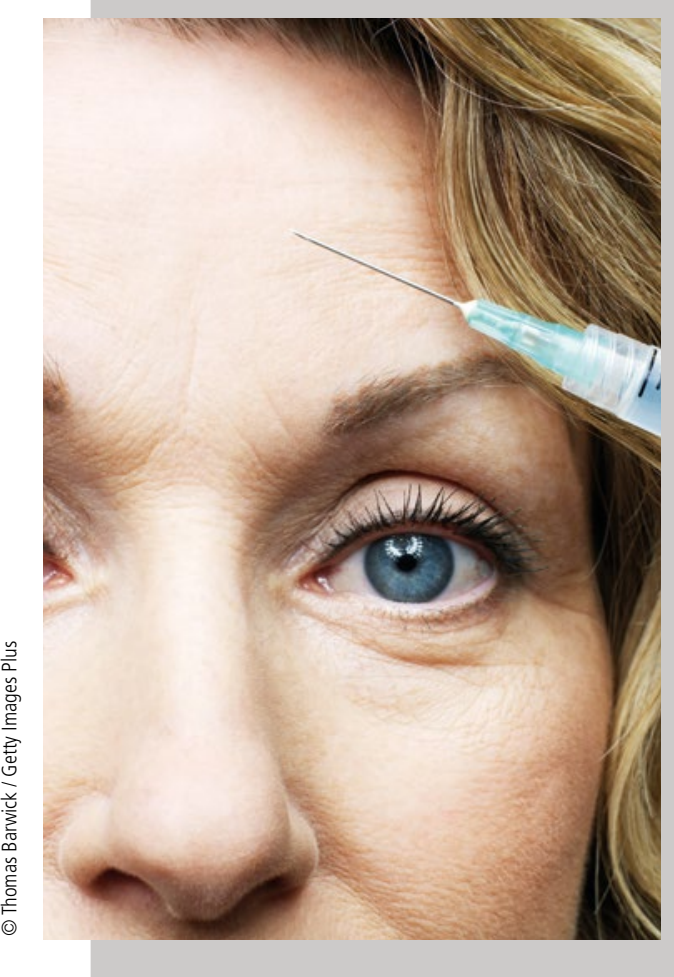

the Committee of Advertising Practice (CAP) Code, even when they are to be administered by a registered healthcare professional. The CAP and Advertising Standards Agency announced that from 1 February 2020, they will use automated technology to identify non-compliant social media posts, which if not removed could result in referral to the Medicines and Healthcare products Regulatory Agency (MHRA) and/or statutory professional regulators such as the GDC.

To aid compliance, the MHRA and CAP have issued new guidance which applies to all social media promotion of botox, including paid-for ads, nonpaid-for marketing posts and 'influencer' marketing. The guidance says there can be no direct references to a prescriptiononly medicine or treatment, whether via a brand name, brand-like name or in the generic, including in images and hashtags or in promotions such as sale packages and competition prizes. Indirect references such as 'anti-wrinkle injections' are also banned.

The organisations instead advise promoting professional consultative services and suggest that advertising 'a consultation for the treatment of lines and wrinkles' may be acceptable. Dermal fillers and other nonPOMs are not covered by the enforcement action or guidance and can still be advertised provided there is no implication that a POM is also available.

Professor Mike Mulcahy, FGDP(UK) Lead on Non-Surgical Facial Aesthetics, said: 'Increasing numbers of dental practices offer injectable cosmetic treatments, and patient demand continues to rise, but many may not be aware of the regulations restricting the advertisement of prescriptiononly medicines and treatments. To avoid the possibility of facing regulatory action, I strongly advise all dentists who administer botox to read the new guidance and immediately review, and if necessary adapt, their social media, websites and other marketing to ensure compliance.'

John Makin, head of the DDU said: 'While the ASA campaign focuses on advertising botulinum toxin injections on social media, the DDU's advice is that dental professionals review all their promotional material including practice websites and leaflets to ensure they comply with the CAP rules, ASA guidance, ethical standards and the law.

'The ASA can take enforcement action for anyone failing to comply with its guidance which could include referral to the Medicines and Healthcare products Regulatory Agency (MHRA) or to the GDC. As a result, it's vital that dental professionals urgently review their advertising materials to ensure compliance.'

\section{New commission calls for dentists to share evidence on alcohol harm}

The Commission on Alcohol Harm has called for evidence as part of its inquiry into alcohol harm and the changes needed to reduce the damage caused by alcohol.

The Commission will hold three oral evidence sessions across the UK later this year, and has launched a call for written evidence, with submissions welcomed before the deadline of 17 February 2020. The Commission is interested in receiving evidence from professionals with experience of the consequences of alcohol harm, particularly those working in dentistry, who often witness the impact of excessive drinking in their work.

With excessive alcohol consumption linked to an array of oral health problems including oral cancer, tooth decay, tooth erosion and accidental dental trauma, dentists and dental nurses can provide expert insight into these areas of alcohol harm.

Professor Sir Ian Gilmore, Chair of the Alcohol Health Alliance UK, said: 'The Commission on Alcohol Harm is an extremely important investigation into how all parts of our society are impacted by our drinking habits.

'We need to have voices representing the wide range of experiences of alcohol harm; including those on the front-line, researchers and those with first-hand experience of alcohol harm. Together, we can build a solid case for change.'

Baroness Finlay of Llandaff, Chair of the Commission on Alcohol Harm, said:
'Alcohol plays a huge part of the everyday lives of many people across the UK, and therefore it is important to examine its impact on our society more closely. We need to understand how our drinking habits affect our own health as well as the way alcohol can affect those around us.

'We welcome the input of those who face the effects of alcohol harm in their professional or personal lives in order to help us make meaningful recommendations on a vision for the future.

To share your views, please submit written evidence to harms_commission@ahauk.org by 17 February 2020 . Visit the AHA website for submission guidelines: http://ahauk.org/ commission-on-alcohol-harm. 